\title{
PVGAMA reticulocyte binding activity: predicting conserved functional regions by natural selection analysis
}

\author{
Luis A. Baquero ${ }^{1 \dagger}$, Darwin A. Moreno-Pérez ${ }^{1,2 \dagger}$, Diego Garzón-Ospina ${ }^{1,2}$, Johanna Forero-Rodríguez ${ }^{1}$
} Heidy D. Ortiz-Suárez ${ }^{1}$ and Manuel A. Patarroyo ${ }^{1,3^{*}}$

\begin{abstract}
Background: Adhesin proteins are used by Plasmodium parasites to bind and invade target cells. Hence, characterising molecules that participate in reticulocyte interaction is key to understanding the molecular basis of Plasmodium vivax invasion. This study focused on predicting functionally restricted regions of the $P$. vivax GPI-anchored micronemal antigen (PVGAMA) and characterising their reticulocyte binding activity.

Results: The pvgama gene was initially found in P. vivax VCG-I strain schizonts. According to the genetic diversity analysis, PVGAMA displayed a size polymorphism very common for antigenic $P$. vivax proteins. Two regions along the antigen sequence were highly conserved among species, having a negative natural selection signal. Interestingly, these regions revealed a functional role regarding preferential target cell adhesion.

Conclusions: To our knowledge, this study describes PVGAMA reticulocyte binding properties for the first time. Conserved functional regions were predicted according to natural selection analysis and their binding ability was confirmed. These findings support the notion that PVGAMA may have an important role in $P$. vivax merozoite adhesion to its target cells.
\end{abstract}

Keywords: Adhesin protein, Plasmodium vivax, Genetic diversity, Conserved functional region, Reticulocyte binding activity

\section{Background}

Plasmodium vivax is a human malaria-causing parasite whose eradication is a priority on the international health agenda [1]. As a strategy for eradicating this species, several research groups have focused their efforts on developing a vaccine, as vaccination has been successful at controlling and eradicating other infectious diseases [2].

It has been suggested that vaccines should consist of key proteins or their fragments used by infectious agents to bind to the target cells [3, 4]. Hence, knowledge of proteins expressed by the parasite at the end of its intraerythrocyte life-cycle, especially those interacting with

\footnotetext{
* Correspondence: mapatarr.fidic@gmail.com

${ }^{\dagger}$ Equal contributors

${ }^{1}$ Molecular Biology and Immunology Department, Fundación Instituto de Inmunología de Colombia (FIDIC), Carrera 50 No. 26-20, Bogotá DC, Colombia

${ }^{3}$ Basic Sciences Department, School of Medicine and Health Sciences, Universidad del Rosario, Carrera 24 No. 63C-69, Bogotá DC, Colombia Full list of author information is available at the end of the article
}

red blood cells (RBC), should prove most suitable as candidate vaccine components.

Current efforts to develop an anti-malarial vaccine have mainly focused on $P$. falciparum, given the availability of robust in vitro culturing techniques for this parasite (currently unavailable for $P$. vivax) which has led to a large-scale identification of genes [5], transcripts [6] and proteins [7]. This information has led to an improved understanding of the molecules involved in $P$. falciparum merozoite invasion of erythrocytes. For example, several adhesin molecules have been described in the apical organelles (rhoptries and micronemes), that facilitate interaction with cell receptors and promote parasite internalisation within the target cell [8]. Several of these proteins are immunogenic and are being evaluated as vaccine candidates in clinical studies [9]. The GPI-anchored micronemal antigen (GAMA) represents one apical protein that has an adhesive role in Plasmodium and Toxoplasma. Plasmodium falciparum GAMA (PfGAMA) binds to human erythrocytes, an interaction 
mediated by its binding region which is located in the amino terminal sequence, and is involved in the sialic acid-independent invasion pathway [10]. On the other hand, GAMA knockouts of T. gondii (TgGAMA) show a reduction in the ability of tachyzoites to attach to the host cell during invasion as well as a delay in the time to death in an in vivo model, suggesting a function during parasite adhesion and invasion [11].

Unfortunately, basic $P$. vivax research has been delayed mainly due to the parasite's preference for invading reticulocytes which are difficult to obtain in the high percentages needed for propagating $P$. vivax in vitro $[12,13]$. However, it has been possible to characterise several molecules forming part of the parasite's selective human reticulocyte invasion route, such as reticulocyte binding proteins (RBPs) $[14,15]$, merozoite surface protein 1 (MSP-1) [16], some proteins from the tryptophanrich antigen (TRAg) family [17] and the recently described rhoptry neck protein 5 (RON5) [18]. Some of these contain specific binding regions that have been identified using several strategies, such as mapping using peptides labelled with radioactive iodine, ELISA, flow cytometry or rosetting assays. However, these methodologies are laborious when large molecules must be analysed. Furthermore, sometimes it is not known whether these regions are polymorphic between isolates, which would be counterproductive for the development of a broadly protective vaccine.

A new strategy has recently been proposed for identifying selection signals and that enables the determination of conserved antigens or those having potential functional regions [19]. Cornejo et al. [20] and GarzónOspina et al. [19] identified natural selection signals in $P$. vivax genes when analysing the sequences of five genomes from different locations [21]. These results were supported by earlier studies, increasing the number of sequences analysed [22-24]. This type of analysis could therefore provide a viable approach for selecting conserved antigens that are subject to functional restrictions. However, no experimental evidence has been produced to support such approach.

Given the importance of conserved functional region prediction and the role of adhesin proteins during host-parasite interaction, and considering the interesting features displayed by GAMA in other apicomplexa, the present study aimed at characterising $P$. vivax VCG-I strain GAMA functional regions by selection signal prediction and then determine the role of such regions in binding to reticulocytes.

\section{Methods}

An approach to GAMA genetic diversity and evolutionary forces

Evolutionary methods compare the non-synonymous mutations rate $\left(\mathrm{d}_{\mathrm{N}}\right.$, mutations altering protein sequences) to the synonymous mutations rate $\left(\mathrm{d}_{\mathrm{S}}\right.$, those encoding the same amino acid) in the search for natural selection signals. Deleterious mutations are usually removed from populations by negative natural selection $\left(\mathrm{d}_{\mathrm{N}}<\mathrm{d}_{\mathrm{S}}\right.$ or $\left.\omega<1\right)$. Regions displaying this kind of selection might have functional/structural importance, maintaining high sequence conservation between species [25]. On the other hand, mutations having an adaptive advantage (or a beneficial role) are fixed in a population by positive natural selection $(\mathrm{dN}>\mathrm{dS}$ or $\omega>1)$. Taking the above into account, functional regions could be predicted by evolutionary approaches [19]. pvgama gene DNA sequences from $6 P$. vivax strains (VCG-I, Sal-I, Brazil-I, India-VII, Mauritania-I and North Korea [21]) and 5 phylogenetically-related species (P. cynomolgi, P. inui, P. fragile, $P$. knowlesi and $P$. coatneyi) [26] were obtained by tblastn (except for VCG-I) from the whole-genome shotgun contigs (wgs) NCBI database for assessing genetic diversity and evolutionary forces regarding GAMA. The MUSCLE algorithm [27] was used to align the sequences and the alignment was manually corrected. Nucleotide diversity per site $(\pi)$ was estimated from the $P$. vivax sequences and the modified Nei-Gojobori method [28] was used to assess natural selection signals by calculating the difference between synonymous and non-synonymous substitution rates $\left(\mathrm{d}_{\mathrm{N}}-\mathrm{d}_{\mathrm{S}}\right)$. Natural selection was also assessed by estimating the difference between synonymous and non-synonymous divergence rates $\left(\mathrm{K}_{\mathrm{N}}-\mathrm{K}_{\mathrm{S}}\right)$ using sequences from $P$. vivax and related species through the modified Nei-Gojobori method and Jukes-Cantor correction [29]. Specific codons under natural selection amongst species were identified using codon-based Bayesian or maximum likelihood approaches (SLAC, FEL, REL [30], MEME [31] and FUBAR [32]), following recombination by the GARD method [33]. Codon-based methods estimate the evolutionary rate $(\omega)$ at each codon using a statistical test to determine whether $\omega$ is significantly different to 1 (neutral evolution). The Branch-site REL algorithm [34] was used to identify lineages under episodic positive selection (selection occasionally having transient periods of adaptive evolution masked by negative selection or neutral evolution). The Datamonkey web server was used to perform these analyses [35].

\section{Primer design, cloning and sequencing}

The Plasmodium vivax gama (pvgama) gene sequence was taken from the PlasmoDB database [36] and scanned for PCR priming sites (Table 1) using Generunner software (version 3.05). Primers were designed to amplify either the entire pvgama gene or several smaller-sized fragments according to the natural selection analysis (Fig. 1). The gDNA (extracted using a Wizard Genomic purification kit; Promega, Madison, USA) and cDNA (synthesised with SuperScript III enzyme (RT+) (Invitrogen, 
Table 1 Primer designed for pvgama gene amplification

\begin{tabular}{|c|c|c|c|c|}
\hline Target & Primer sequence $\left(5^{\prime}-3^{\prime}\right)^{a}$ & MT $\left({ }^{\circ} \mathrm{C}\right)$ & Product size (bp) & aa position \\
\hline pvgama & $\begin{array}{l}\text { Fwd: ATGAAGTGCAACGCCTCC } \\
\text { Rev: AAAAATGAATAGGAGCAACG }\end{array}$ & 58 & 2313 & 1 to 771 \\
\hline pvgama - Nt & $\begin{array}{l}\text { Fwd: ATACGGAATGGAAACAACC } \\
\text { Rev: AGTCGGTCGTTATTCTCG }\end{array}$ & & 1284 & 22 to 449 \\
\hline pvgama -Ct & $\begin{array}{l}\text { Fwd: CTGCTCAAGAACACGAAC } \\
\text { Rev: GCTTCCACTCTGCAATTC }\end{array}$ & & 948 & 434 to 749 \\
\hline pvgama -CR1 & $\begin{array}{l}\text { Fwd: GACGATCATCTGTGTTCAAAAA } \\
\text { Rev: GACCTCATTITTGACTTCTC }\end{array}$ & 60 & 666 & 87 to 308 \\
\hline pvgama -VR1 & $\begin{array}{l}\text { Fwd: GGCGCCTTCCTGCAGTC } \\
\text { Rev: CATTAACATGGTGTTGTCGCT }\end{array}$ & & 438 & 330 to 475 \\
\hline pvgama -CR2 & $\begin{array}{l}\text { Fwd: CAGGCGGCCATCTTACTAA } \\
\text { Rev: GCTCCCGTTGACGCCCTT }\end{array}$ & & 321 & 482 to 588 \\
\hline pvgama -VR2 & $\begin{array}{l}\text { Fwd: GCCGCAAACGCAGACGCC } \\
\text { Rev: GTTTGCCGAGAAGCTTCCAC }\end{array}$ & & 384 & 626 to 753 \\
\hline
\end{tabular}

Abbreviations: $N t$ and $C t$ amino and carboxyl terminal; $C R$ conserved region, $V R$ variable region; Fwd forward, Rev reverse, $M T$ melting temperature, bp base pair, ac amino acid

'Protein's expression start codon was included in forward primer's 5 ' end

Carlsbad, USA) samples from $P$. vivax VCG-I strain schizont-stage enriched parasites (propagated and obtained as previously described [37, 38]) were used as template in $25 \mu \mathrm{l}$ PCR reactions containing $1 \times$ KAPA HiFi HotStart ReadyMix (KAPA Biosystems, Woburn, MA, USA), $0.3 \mu \mathrm{M}$ primers and DNAse-free water. Temperature cycling for PCR involved a denaturing step of $95{ }^{\circ} \mathrm{C}$ for $5 \mathrm{~min}$, followed by 35 cycles of $98{ }^{\circ} \mathrm{C}$ for $20 \mathrm{~s}$, Tm ${ }^{\circ} \mathrm{C}$ (Table 1) for $15 \mathrm{~s}$ and $72{ }^{\circ} \mathrm{C}$ for $30 \mathrm{~s}$ or $1 \mathrm{~min}$ and $30 \mathrm{~s}$ depending on product size. A Wizard PCR preps kit (Promega) was used for purifying amplicons obtained from PCR with the RT+ and gDNA samples, once quality had been evaluated on agarose gel. Purified products were ligated to the pEXP5 CT/TOPO expression vector or pGEM (Promega) (for the gene obtained from gDNA) and transformed in TOP10 E. coli cells (Invitrogen). Several clones obtained from independent PCR reactions were grown for purifying the plasmid using an UltraClean mini plasmid prep purification kit (MO BIO Laboratories, California, USA). Insert integrity and correct orientation were then confirmed by sequencing, using an $\mathrm{ABI}-3730 \mathrm{XL}$ sequencer (MACROGEN, Seoul, South Korea). ClustalW (NPS@) software was used for comparing gene sequences from Sal-I reference strain and the primate-adapted VCG-I strain [39]. The pvgama gene sequence from $P$. vivax VCG-I strain was deposited in NCBI under accession number KT248546.

\section{Recombinant protein expression}

The pEXP-pvgama recombinant plasmids were transformed in E. coli BL21-DE3 (Invitrogen), according to the manufacturer's recommendations. Cells were grown

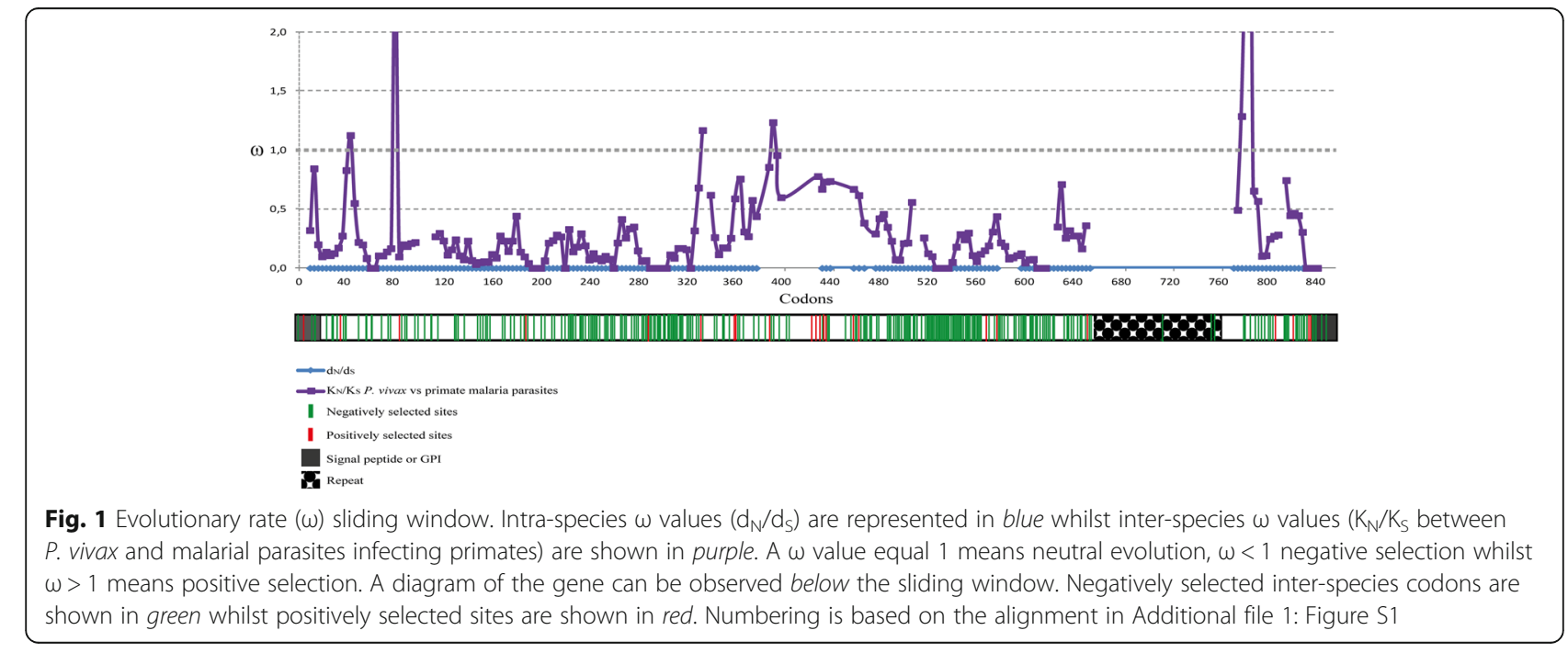


overnight at $37{ }^{\circ} \mathrm{C}$ in $50 \mathrm{ml}$ Luria Bertani (LB) medium containing $100 \mu \mathrm{g} / \mathrm{ml}$ ampicillin using a Lab-line Incubator Shaker. The initial inoculum was then seeded in $1 \mathrm{lof} \mathrm{LB}$ with ampicillin $(100 \mu \mathrm{g} / \mathrm{ml})$ and left to grow at $37{ }^{\circ} \mathrm{C}$ with shaking at $\sim 300 \times \mathrm{rpm}$ until reaching 0.5 $\mathrm{OD}_{600}$. The culture was incubated on ice for $30 \mathrm{~min}$ and then IPTG $1 \mathrm{mM}$ was used to induce expression by incubation for $16 \mathrm{~h}$ at room temperature (RT) with shaking at $\sim 200 \times \mathrm{rpm}$. The culture was then spun at $2400 \times g$ for $20 \mathrm{~min}$ and the pellet was collected for extraction of the recombinant protein.

\section{Denaturing extraction}

The cell pellet obtained from E. coli expressing $P \nu$ GAMA-Nt and $P \nu$ GAMA-Ct fragments was homogenised in denaturing extraction buffer (DEB) (6 M urea, $10 \mathrm{mM}$ Tris, $100 \mathrm{mM} \mathrm{NaH}_{2} \mathrm{PO}_{4}$ and $20 \mathrm{mM}$ imidazole) containing the SIGMAFAST protease inhibitor cocktail (Sigma-Aldrich, St. Louis, USA) and then lysed by incubating with $0.1 \mathrm{mg} / \mathrm{ml}$ lysozyme overnight at $4{ }^{\circ} \mathrm{C}$ at $10 \times$ rpm using a tube rotator (Fisher Scientific, Waltham, USA). The supernatant was collected by spinning at $16,000 \times g$ for $1 \mathrm{~h}$.

\section{Native extraction}

PvGAMA-CR1, PvGAMA-VR1, PvGAMA-CR2 and PvGAMA-VR2 were extracted using a method for obtaining the molecules in native conditions with the respective positive and negative controls (region II and III/IV from the Duffy binding protein, DBP) (unpublished data). Briefly, the pellet was frozen/thawed for 3 cycles and then homogenised in native extraction buffer (NEB) (50 mM Tris, $300 \mathrm{mM} \mathrm{NaCl}, 25 \mathrm{mM}$ imidazole, $0.1 \mathrm{mM}$ EGTA and $0.25 \%$ Tween-20, $\mathrm{pH}$ 8.0). The mixture was incubated for $1 \mathrm{~h}$ at $4{ }^{\circ} \mathrm{C}$ at $10 \times \mathrm{rpm}$ and the supernatant was collected by spinning at $16,000 \times g$ for $1 \mathrm{~h}$.

\section{Protein purification}

Total lysate supernatant was incubated with $\mathrm{Ni}^{+2}$-NTA resin (Qiagen, Valencia, CA, USA) for purifying the proteins by solid-phase affinity chromatography, once protein expression had been verified by western blot. Briefly, the resin was pre-equilibrated with the respective buffer used for extracting proteins and then incubated with the E. coli lysate overnight at $4{ }^{\circ} \mathrm{C}$. The proteinresin mixture was placed on a column and then weakly bound proteins were eluted by washing with $20 \mathrm{ml}$ buffer containing $0.1 \%$ Triton X-114 followed by $50 \mathrm{ml}$ of the same buffer without detergent. The proteins extracted in denaturing conditions were dialysed on the column by passing $20 \mathrm{ml}$ DEB with urea in descending concentrations $(6 \mathrm{M}, 3 \mathrm{M}, 1.5 \mathrm{M}, 0.75 \mathrm{M}$ and PBS). Bound proteins were then eluted with PBS containing imidazole at increasing concentrations $(50 \mathrm{mM}$ to
$500 \mathrm{mM}$ ) in $3 \mathrm{ml}$ fractions; those having a single band (confirmed on 12\% SDS-PAGE by Coomassie blue staining and by western blot using anti-polyhistidine antibodies) were pooled and dialysed extensively in PBS, pH 7.2. A micro BCA protein assay kit (Thermo Scientific, Rockford, USA) was used for quantifying each protein, using the bovine serum albumin (BSA) curve as reference.

\section{Peptide synthesis}

One 6 histidine peptide was synthesised according to a previously-established methodology [40], polymerised, lyophilised and characterised by RP-HPLC and MALDITOF MS. The peptide was homogenised in PBS and then stored at $-20{ }^{\circ} \mathrm{C}$ until use.

\section{Blood sample collection and processing}

Individuals with a clinical history of $P$. vivax (37 subjects) or P. falciparum (30 subjects) malaria, aged 18 to 50 yearold and living in malaria-endemic areas of Colombia (Chocó, Nariño, Córdoba, Vichada and Guaviare) were selected for this study. Sera from healthy individuals (16 adult subjects) who had never been affected by the disease and who were living in non-endemic areas were used as negative controls. The blood samples were collected in BD Vacutainer tubes without anticoagulant by personnel from the Fundación Instituto de Inmunología de Colombia (FIDIC) from October 2006 to March 2011 (for P. vivax) and June to October 1993 (for P. falciparum) and stored at $4{ }^{\circ} \mathrm{C}$ until transport. Samples were then transported to Bogotá for processing. Total blood was spun at $5000 \times g$ for $5 \mathrm{~min}$ and the serum was then recovered and stored at $-80{ }^{\circ} \mathrm{C}$ in FIDIC serum bank (to date).

\section{Enzyme-linked immunosorbent assay (ELISA)}

$P \nu$ GAMA antigenicity was evaluated in triplicate using serum from patients who had suffered episodes of $P$. vivax or $P$. falciparum infection. Briefly, 96-well polysorb plates were covered with $1 \mu \mathrm{g} r P v$ GAMA-Nt, or rPvGAMA-Ct, overnight at $4{ }^{\circ} \mathrm{C}$ and then incubated at $37{ }^{\circ} \mathrm{C}$ for $1 \mathrm{~h}$. The dishes were blocked with $200 \mu \mathrm{l} 5 \%$ skimmed milk - PBS-0.05\% Tween for $1 \mathrm{~h}$ at $37{ }^{\circ} \mathrm{C}$. Antibody reactivity against the recombinant protein was evaluated by incubating the plates with 1:100 dilution of each human serum in 5\% skimmed milk - PBS$0.05 \%$ Tween for $1 \mathrm{~h}$ at $37{ }^{\circ} \mathrm{C}$. The dishes were incubated with peroxidase-coupled goat anti-human IgG monoclonal secondary antibody $(1: 10,000)$ (Catalogue $1222 \mathrm{H}, \mathrm{ICN})$ diluted in 5\% skimmed milk - PBS-0.05\% Tween for $1 \mathrm{~h}$ at $37{ }^{\circ} \mathrm{C}$ and then a peroxidase substrate solution (KPL Laboratories, Gaithersburg, MD, USA) was added to reveal the reaction, according to the manufacturer's recommendations. Optical density (OD) at $620 \mathrm{~nm}$ (detected by MJ ELISA Multiskan Reader) was 
calculated by subtracting the OD value obtained from the control well value (no antigen). The cut-off value for evaluating the positivity threshold was determined by taking the average of the OD plus twice the standard deviation $( \pm 2 \mathrm{SD})$ of healthy individuals' sera reactivity.

\section{Cord blood sample processing}

The newborn umbilical cord blood samples used in this research were collected by personnel from the Hemocentro Distrital (Bogotá) and then processed by SEPAX Cell Processing System (Biosafe, Eysins, Switzerland) to reduce nucleated cells, according to the manufacturer's recommendations. The samples were stored at $4{ }^{\circ} \mathrm{C}$ and Duffy antigen receptor for chemokines (DARC) presence was determined by agglutination assay using antibodies directed against the molecule's Fya or Fyb fraction. The percentage of nucleated cells was scored in 20 fields at 100x magnification using Wright's stain before carrying out the binding assay.

\section{Cell binding assay}

Reticulocyte binding was tested in triplicate by flow cytometry and using the total cells from cord blood sample (Fya $\mathrm{Fyb}^{+}$phenotype). Briefly, $5 \mu \mathrm{l}$ samples were incubated with $25 \mu \mathrm{g}$ of each recombinant protein (PvGAMA-CR1, PvGAMA-VR1, $P \nu$ GAMA-CR2 and PvGAMA-VR2) for $16 \mathrm{~h}$ at $4{ }^{\circ} \mathrm{C}$ at $4 \times \mathrm{rpm}$. Twenty-five $\mu \mathrm{g}$ of DBP region II and III/IV were used as positive and negative controls, respectively. The 6 histidine peptide was also used as control once the recombinant proteins contained a 6-histidine tag. A binding inhibition assay was also performed by incubating PvGAMA conserved recombinant proteins (CR1 and CR2) with a mixture of human sera (1:10 dilution) for $1 \mathrm{~h}$ at $4{ }^{\circ} \mathrm{C}$ before putting them in contact with cells. The samples were then incubated with mouse anti-His-PE monoclonal antibody (1:40 dilution) (MACSmolecular-Miltenyi Biotec, San Diego, CA, USA) for $30 \mathrm{~min}$ in the dark after washing with $1 \%$ BSA-PBS solution $(\mathrm{v} / \mathrm{v})$. White cells and reticulocytes were stained by incubating with anti-CD45 APC clone 2D1 (1:80 dilution) (Becton Dickinson, Franklin Lakes, NJ, USA) and anti-CD71 APC-H7 clone M-A712 (1:80 dilution) (Becton Dickinson) monoclonal antibodies for $20 \mathrm{~min}$ at RT. Subsequently, reticulocyte (CD71 + $\mathrm{CD} 45-\mathrm{PE}+$ ) and mature erythrocyte (CD71-CD45-PE+) binding was quantified by analysing 1 million events using a FACSCanto II cytometer (BD, San Diego, CA, USA) and Flowjo V10 software. PE signal intensity in the reticulocyte population was evaluated regarding CD71 signal to determine $\mathrm{CD} 71$ low $\left(\mathrm{CD} 71^{\mathrm{lo}}\right)$ and high $\left(\mathrm{CD} 71^{\mathrm{hi}}\right)$ cells.

\section{Statistical analysis}

Mean values and standard deviations (SD) were calculated from the measurements of three independent experiments. Statistical significance was assessed by comparing means using a 0.05 significance level for testing a stated hypothesis. Student's $t$-test and analysis of variance (ANOVA) were used for comparing the means of each experimental group to those for control. Tukey's multiple comparison test was used for multiple comparison of experimental group means to those for control. GradhPad Software (San Diego, CA) was used for all statistical analysis.

\section{Results}

\section{PvGAMA genetic diversity and selection signals}

Pvgama sequences were obtained from genomes of 5 different strains from different geographical regions (North Korea, Brazil, Mauritania and India). These were aligned with the VCG-I strain sequence and orthologous sequences from 5 phylogenetically-related species. The alignment revealed a size polymorphism in pvgama due to the $[\mathrm{C} / \mathrm{T}] \mathrm{C}[\mathrm{G} / \mathrm{C}] \mathrm{C}[\mathrm{A} / \mathrm{T}] \mathrm{AA}[\mathrm{C} / \mathrm{T}][\mathrm{C} / \mathrm{G}][\mathrm{A} /$ $\mathrm{G} / \mathrm{C}][\mathrm{G} / \mathrm{A}] \mathrm{AC}[\mathrm{G} / \mathrm{C} / \mathrm{A}]$ repeat which was not present in $P$. cynomolgi, $P$. inui, $P$. fragile, $P$. knowlesi or $P$. coatneyi (Additional file 1: Figure S1). Regarding $P$. vivax, 5 segregating sites and $\pi=0.0008$ were observed.

No significant values were found when evaluating synonymous and non-synonymous substitution rates $\left(\mathrm{d}_{\mathrm{N}}-\mathrm{d}_{\mathrm{S}}=-0.001(0.001), P>0.1\right)$. However, synonymous divergence was greater than non-synonymous divergence $(P<0.0001)$ when comparing pvgama sequences to each related species: $\mathrm{K}_{\mathrm{N}}-\mathrm{K}_{\mathrm{S}} P$. vivax $/ P$. cynomolg $i=-0.041$ (0.006); $\mathrm{K}_{\mathrm{N}}-\mathrm{K}_{\mathrm{S}}$ P. vivax/P. inui $=-0.062(0.008) ; \mathrm{K}_{\mathrm{N}}-\mathrm{K}_{\mathrm{S}} P$. vivax/P. fragile $=-0.030(0.006) ; \mathrm{K}_{\mathrm{N}}-\mathrm{K}_{\mathrm{S}} P$. vivax/P. knowlesi $=-0.072(0.009) ; \mathrm{K}_{\mathrm{N}}-\mathrm{K}_{\mathrm{S}} P$. vivax $/ P$. coatneyi $=-0.049$ (0.007). The evolutionary rate $\omega(\mathrm{dN} / \mathrm{dS}$ and $\mathrm{KN} / \mathrm{KS})$ sliding window showed that two highly conserved regions amongst species (codons $80-320$ and 514-624) might be under negative selection $(\omega<0.5)$. Furthermore, 308 negatively-selected codons were observed amongst species (Fig. 1); a lot of them were in the conserved regions. The Branch-site REL algorithm identified episodic positive selection signals in the lineages giving rise to $P$. knowlesi and $P$. coatneyi as well as the lineage formed by $P$. cynomolgi and $P$. fragile (Additional file 2: Figure S2). 22 sites showed evidence of positive selection amongst species (Fig. 1).

\section{Antigenic response was directed against the GAMA carboxyl fragment}

Based on the polymorphism analysis results, it was hypothesised that the carboxyl region was more antigenic than the amino one by the presence of the repetitive region. Hence, rPvGAMA-Nt and $\mathrm{r} P \nu$ GAMA-Ct antigenicity (obtained recombinantly; Additional file 3: Figure S3a, b) was evaluated using sera from 37 patients suffering of $P$. vivax malaria and sera from people who had never suffered the disease. rPvGAMA-Nt reacted 
positively with $64.8 \%$ of the sera in screening ( 0.26 cut-off point) whilst $67.5 \%$ of them recognised $\mathrm{rP \nu GAMA-Ct}$ (0.47 cut-off point). These data agreed with a study of the profile of the humoral immune response for $P$. vivax in which $\mathrm{r} P \nu \mathrm{GAMA}$ was recognised by $54.5 \%$ of the sera used in the array [41]. The statistical test for the assay with r $P \nu$ GAMA-Nt gave a significant difference between the means $(m)$ of the groups (ANOVA: $F_{(1,41)}=4.73, P=$ 0.035; $m=0.38$ for the group of infected patients and $m=$ 0.12 for the control group). Likewise, there was a significant difference between the means of the groups (ANOVA: $F_{(1,41)}=14.75, P=0.0001 ; m=0.67$ for the group of infected patients and $m=0.14$ for the control group) when $\mathrm{r} P v$ GAMA-Ct was detected by human sera (Fig. 2a). There was also a statistically significant difference when analysing the means of recognition for rPvGAMA-Nt and $\mathrm{rPvGAMA-Ct}$ (ANOVA: $F_{(1,72)}=$ $16.01, P=0.0002)$. Taking into account that the response was higher against PvGAMA-Ct, it was decided to confirm whether the antibodies generated during $P$. falciparum natural infection were able to detect this fragment. No significant difference (ANOVA: $F_{(1,38)}=0.036, P=$ 0.850 ) was seen for $P \nu$ GAMA-Ct recognition by these sera (Fig. 2b). The significant reactivity against the recombinants by $P$. vivax-infected individuals' sera indicated that the protein could trigger an antigenic response during natural infection, this being higher and species-specific against the $P \nu$ GAMA carboxyl region.

\section{PvGAMA bound to human reticulocytes}

Red blood cell samples having the Fya ${ }^{-} \mathrm{Fyb}^{+}$phenotype $($ Duffy + ) taken from umbilical cord blood were incubated with conserved (CR1 and CR2) and variable (VR1 and VR2) regions extracted and purified in their soluble form (Additional file 3: Figure S3c), predicted by natural selection analysis and then evaluated by flow cytometry to quantify the protein-cell interaction. The percentage of each recombinant binding to erythrocytes was calculated using the gating strategy described in Additional file 4: Figure S4, which enabled selecting the mature (CD71-CD45-) or immature $(\mathrm{CD} 71+\mathrm{CD} 45-)$ cell population to which a target protein was bound (labelled with anti-His PE antibody). All recombinant proteins had a curve shift when the PE signal was compared to control (cells not incubated with recombinant proteins) in the histogram (Fig. 3). Interestingly, the GAMA fragments bound to reticulocytes to a much higher percentage compared to mature erythrocytes (CR1: $t$-test: $t_{(4)}=24.9, P=$ 0.0001; VR1: $t$-test: $t_{(4)}=9.02, P=0.001$; CR2: $t$-test: $t_{(4)}=12.4, \quad P=0.0001 ; \quad$ VR2: $t$-test: $t_{(4)}=24.8, \quad P=$ 0.0001) (Fig. 4a). The conserved regions showed highest interaction with the reticulocytes compared to negative binding controls (ANOVA-Tukey: $F_{(6,12)}=$ $72.64, P<0.0001)$. CR2 recombinant protein bound to $10.11 \%(\mathrm{SD}=1.33)$ of target cells, which was very similar to the positive control $(m \pm \mathrm{SD}=11.8 \pm 1.15)$ $(P>0.189)$, whilst CR1 were able to bind to $6.36 \%$ $(\mathrm{SD}=0.30)$ of the cells (Fig. 4a). Regarding PvGAMA variable regions, VR1 was able to bind to $3.08 \%$ ( $\mathrm{SD}=$ 0.54 ) of the reticulocytes whilst VR2 bound $5.64 \%$ ( $\mathrm{SD}=$ 0.37). CR1, CR2 and VR2 fragments had the highest interaction with $\mathrm{CD} 71^{\mathrm{hi}}$ reticulocytes when binding percentages were analysed as a function of CD71 APC-H7 signal (CR1: $t$-test: $t_{(4)}=7.32, P=0.002$; CR2: $t$-test: $t_{(4)}=16.04$, $P=0.0001$; VR2: $t$-test: $\left.t_{(4)}=3.71, P=0.021\right)$, unlike VR1 and DBP-RII (VR1: $t$-test: $t_{(4)}=1.52, P=0.202$; DBP-RII: $t$-test: $t_{(4)}=0.19, P=0.853$ ) (as previously found [42])
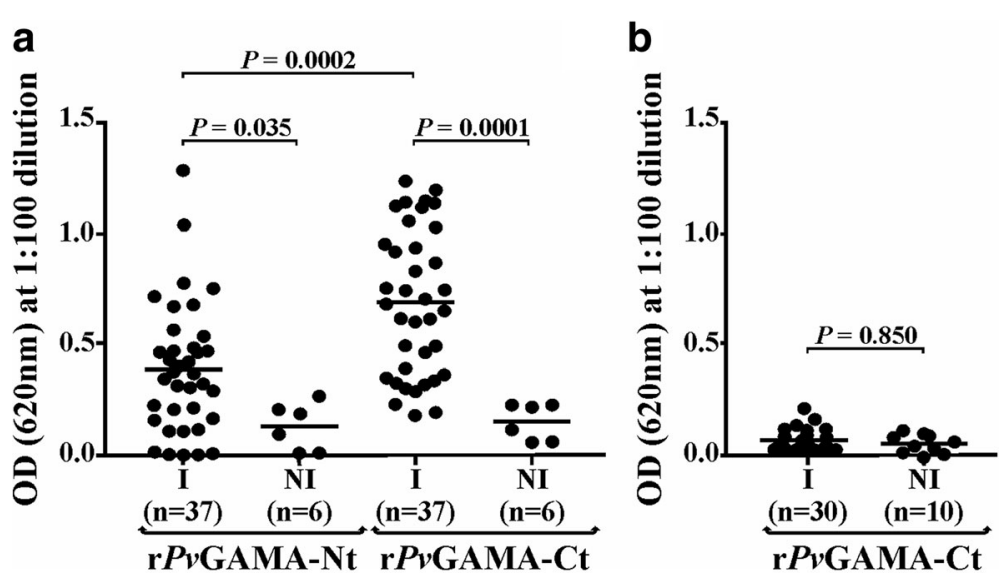

Fig. 2 PVGAMA antigenicity during natural malaria infection. The dot plot shows OD distribution (Y-axis) for detecting rPVGAMA-Nt or rPVGAMA-Ct by P. vivax (a) or rPvGAMA-CT by P. falciparum (b) infected (I) and non-infected (NI) patients' sera (X-axis). rPvGAMA-Nt: infected individuals $n=37$, $m \pm \mathrm{SD}=0.38 \pm 0.29$; control individuals $n=6, m \pm \mathrm{SD}=0.12 \pm 0.1$. rPvGAMA-Ct: infected individuals $n=37, m \pm \mathrm{SD}=0.67 \pm 0.32$; control individuals $n=6, m \pm S D=0.14 \pm 0.08$. rPVGAMA-Ct recognised by $P$. falciparum infected patients' sera: infected individuals $n=30, m \pm S D=0.06 \pm 0.04 ;$ control individuals $n=10, m \pm S D=0.06 \pm 0.03$ 
a

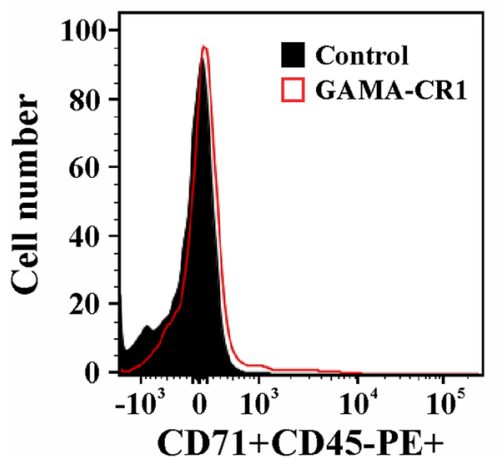

C

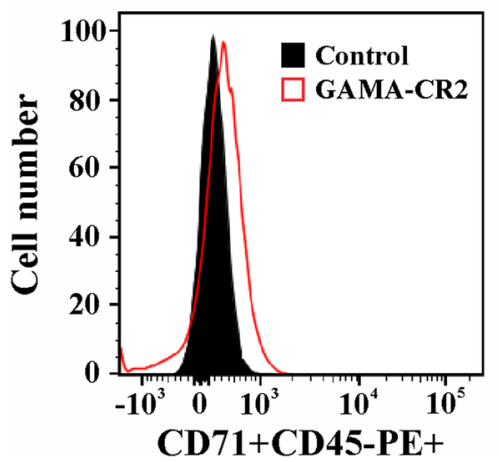

b

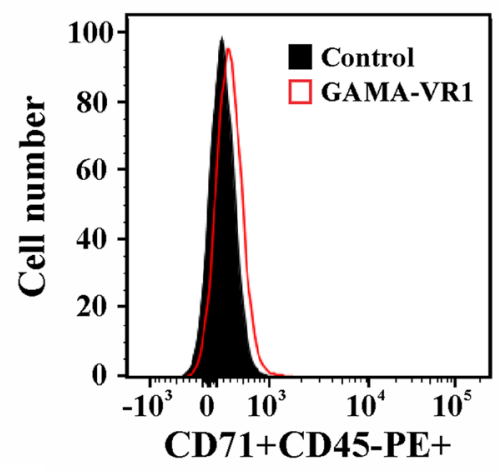

d

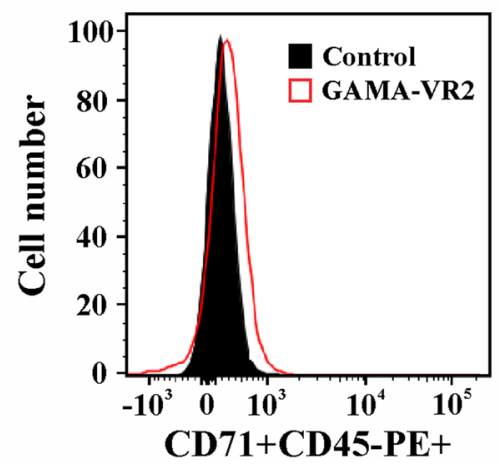

Fig. 3 Flow cytometry analysis. Histograms of conserved (a and $\mathbf{c}$ ) and variable (b and $\mathbf{d}$ ) GAMA fragments compared to control (cells not incubated with the protein). Each figure is representative from three independent experiments

(Fig. 4b). These findings suggested that GAMA in P. vivax has a functional role in preferential interaction with human reticulocytes.

Natural antibodies did not affect PvGAMA binding activity A cytometry adhesion inhibition assay was performed with sera from individuals suffering $P$. vivax malaria to determine whether the antibodies produced during natural infection could inhibit functional conserved regions (CR1 and CR2) interaction with reticulocytes. Figure 4c shows that conserved recombinant proteins pre-incubated with human sera were able to bind to target cells (CR1: $m \pm$ $\mathrm{SD}=6.21 \pm 0.27$; $\mathrm{CR} 2: m \pm \mathrm{SD}=9.83 \pm 0.09)$, giving a similar percentage to that for controls (CR1: $m \pm \mathrm{SD}=6.5 \pm$ 0.08; CR2: $m \pm \mathrm{SD}=10.01 \pm 0.95)\left(\mathrm{CR} 1: t\right.$-test: $t_{(3)}=0.55$, $P=0.617 ; C R 2$ : $t$-test: $\left.t_{(4)}=0.37, P=0.730\right)$, suggesting that the immune response was directed against regions which are not implicated in cell binding.
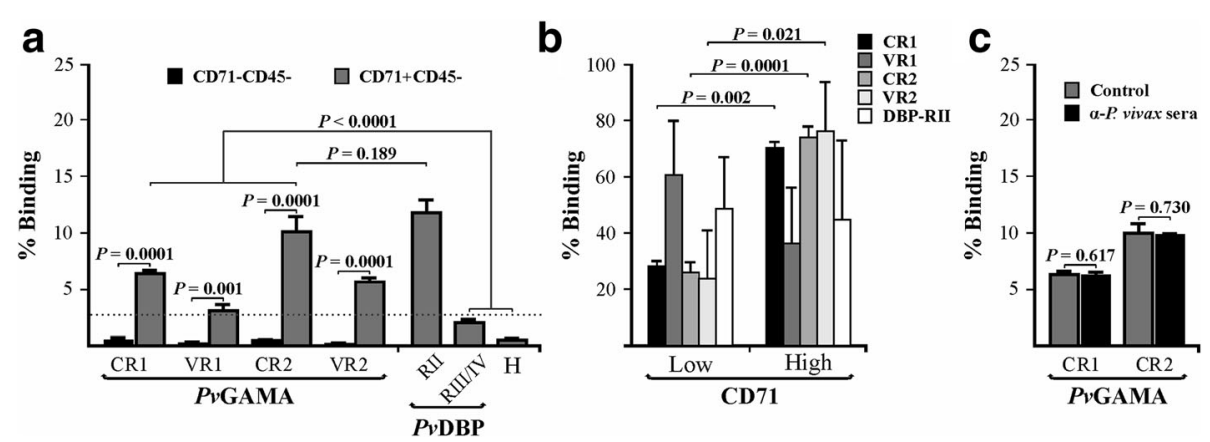

Fig. 4 PVGAMA human reticulocyte binding activity. Flow cytometry analysis showing the recombinant binding percentage to CD71-CD45and CD71 + CD45- cells (a) and regarding CD71-APCH7 signal (only for CD71 + CD45- cells) (b). Positive (DBP-RII) and negative (DBP-RIII/IV and $\mathrm{H}$ (6 histidine peptide)) binding controls are also shown. c CR1 and CR2 reticulocyte binding inhibition assay using human sera (a-P. vivax sera). Binding percentage in both analyses were expressed as mean \pm SD of three independent experiments 


\section{Discussion}

Merozoite invasion of erythrocytes involves the participation of several parasite molecules expressed at the end of the intra-erythrocyte lifecycle, mainly those contained in the apical organelles, such as the rhoptries and micronemes [8]. Only a few of these molecules possessing a reticulocyte binding role in $P$. vivax have been identified and their binding domains mapped, suggesting an urgent need for performing further studies to supplement current knowledge on $P$. vivax adhesins. This will improve our understanding of the molecular basis of parasite invasion of reticulocytes. This study aimed at using natural selection analysis for identifying GAMA functional regions playing a potential role in reticulocyte binding.

According to the phylogenetic analysis, a repeat region (RR) localised between amino acids 591 and 695 consisting of residues $[\mathrm{A} / \mathrm{L}] \mathrm{AN}[\mathrm{A} / \mathrm{G}][\mathrm{N} / \mathrm{D}]$ was predicted. This $\mathrm{RR}$ was common in different $P$. vivax strains but not in phylogenetically-related species (Additional file 1: Figure S1). This characteristic has been found in several $P$. vivax antigens described in the $P$. vivax VCG-I strain located on the parasite surface (Pv12 [12], ARP [43]) or in the apical pole (Pv34 [44], RON1 [45], RON2 [46] and RON4 [47, 48]). DNA sequences from different $P$. vivax strains and phylogenetically-related species were thus compared to ascertain whether gama gene diversity has been modulated by immune pressure. Evidence of episodic positive selection was found in some parasite lineages (Additional file 2: Figure S2). As shown for other antigens [49-51], the episodic selection found in GAMA could be the outcome of adaptation to different hosts during malaria-primate evolution [50, 51]. Therefore, the insertions found in $P$. vivax could be an adaptation of the species to humans since the RR in malaria are associated with evasion of the host's immune response, making such response become directed against functionally unimportant regions [52, 53]. This hypothesis was supported by the fact that $\mathrm{r} P \nu \mathrm{GAMA}-\mathrm{Ct}$ (where the $\mathrm{RR}$ is located) can trigger a species-specific immune response (Fig. 2) which did not inhibit CR2 binding activity to reticulocytes (Fig. 4c).

Polymorphic regions induce high levels of strainspecific antibodies (allele specific) whilst conserved regions (directly implicated in interaction with cell receptors) are usually non-antigenic [54]. Therefore, the immune response must be directed against conserved regions to avoid different parasite strains evading immunity, thereby reducing vaccine efficacy. According to the selection signal identification strategy, low genetic diversity was found in the GAMA-encoding gene, comparable to that observed in $\operatorname{msp} 4[55,56]$, msp7A/7 K/7 F/7 L [57, 58], msp8 [59], msp10 [57, 59], pv12, pv38 [22, 24], pv41 [23, 24], rap1/2 [60] and ron4
[48] which seem involved in host cell invasion. Despite the lack of statistically significant values for $d_{N}-d_{S}$ difference, $K_{S}$ divergence amongst species was greater than $K_{N}$, suggesting negative selection. Many codons were found to be experiencing negative selection which probably plays an important role in GAMA evolution. Two regions along the antigen were highly conserved amongst species, giving $\mathrm{a}<0.5$ evolutionary rate $(\omega)$ (Fig. 1 ).

Given the polymorphism and selection analysis, it was decided to determine PvGAMA conserved and variable region interaction with reticulocytes to validate the in silico prediction of functional regions (Figs. 3 and 4) and elucidate the protein's function. A reticulocyte sample having a Duffy positive phenotype was used, given that $P \nu$ GAMA reportedly has a binding role regardless of such antigen's expression [61]. Unlike Cheng and his group, the anti-CD71 monoclonal antibody was included for identifying GAMA regions' preference for immature reticulocyte binding as $P$. vivax merozoites have tropism for this cell type (characterised by the expression of the CD71 receptor [62]). Given that the CD71 marker is also present in activated lymphocytes, a nucleated cell depleted umbilical cord blood sample was used. The anti-CD45 was also included to totally exclude the lymphocytes from the analysis once the Wright staining revealed $0.4 \%$ of such cells (also confirmed by cytometry analysis) (Additional file 4: Figure S4). It was also confirmed that there was no difference in reticulocyte percentage by incubating the samples for 4 and $16 \mathrm{~h}$ at $4{ }^{\circ} \mathrm{C}$ (4 h: $m \pm \mathrm{SD}=1.24 \pm 0.27 ; 16 \mathrm{~h}: m \pm \mathrm{SD}=1.31 \pm 0.07)(t$ test: $\left.t_{(2)}=0.32, P>0.777\right)$. However, it was decided to use a prolonged incubation time to enable complete proteincell interaction.

It was found that all PvGAMA fragments bound to mature erythrocytes (CD71-CD45-) though to a lesser extent compared to reticulocytes (CD71 + CD45-) (Fig. 4a), thereby supporting the fact that the protein preferentially interacts with the latter cell type. The conserved fragment located in the carboxyl region (CR2) had higher reticulocyte binding than the amino one (CR1) (Fig. 4a) coinciding with that shown recently for PvGAMA where this fragment [F2 (aa 345 to 589) or F7 (408 to 589) regions in that study] showed higher rosetting activity, unlike the F1 region (aa 22 to 344) (amino fragment) [61]. Interestingly, CR1 and CR2 had higher CD71 ${ }^{\text {hi }}$ reticulocyte binding percentages than to CD71 ${ }^{\text {lo }}$ (Fig. $4 \mathrm{~b}$ ), suggesting that GAMA mainly binds to such cell type's most immature stage. It has been reported that some reticulocytes' integral membrane components decrease as cells mature [63]. Therefore, the findings found here suggest that $P v$ GAMA receptor is less abundant in $C D 71^{\text {lo }}$ cells unlike $C D 71^{\text {hi }}$, as a consequence of cell maturation. The fact that more than $69 \%$ of the CD71 + CD45- cells were CD71 ${ }^{\text {lo }}(m \pm$ 
$\mathrm{SD}=69.3 \pm 3.3) \quad$ can be the explanation of why PvGAMA fragment binding to $100 \%$ of the CD71+ reticulocytes was not found (Fig. 4a). It has been observed that several $P$. vivax proteins, such as DBP [64], MSP-1 [16], RBP1 [14], the erythrocyte binding protein (EBP) [42], RBP1a, RBP1b [65] and RBP2 [15], have preferential reticulocyte binding activity, being the RBPs particularly important in parasite cell selection. Taking the results obtained here into account, it can be suggested that $P$. vivax target cell selection is not only governed by the RBPs but other ligands are also taking place in this process, such as DBP, MSP-1, EBP and now PvGAMA.

Immunoreactive proteins are considered potential candidates for developing a vaccine as it has been seen that an immune response induced during infection is related to naturally-acquired immunity [66]. Antigenicity is thus one of the classical parameters for selecting molecules when developing a vaccine. Although there was an immune response against $P \nu$ GAMA (Fig. 2), this was not sufficient to inhibit the conserved regions binding to reticulocytes (Fig. 4c). It has been observed that P. falciparum proteins' conserved regions (implicated in target cell binding) cannot trigger an immune response when used as vaccine candidates in the Aotus model whilst non-conserved ones trigger protective responses upon parasite challenge but those are strainspecific [54]. Accordingly, the PvGAMA antibodies produced/induced during natural $P$. vivax infection were directed against immunodominant epitopes which are unimportant in binding activity. Bearing in mind that functional regions usually evolve more slowly and that natural negative selection tends to keep these regions conserved amongst species [25], our experimental findings suggested that CR1 and CR2 located between residues $80-320$ ( $40 \%$ of negatively selected sites) and $514-624$ (64.5\% of negatively selected sites) are functionally/structurally restricted and that vaccine design should thus be focused on them.

\section{Conclusions}

To our knowledge, this study described PvGAMA reticulocyte binding properties for the first time. The PvGAMA antigenic response was principally directed against its carboxyl fragment which comprises by a repetitive region. On the other hand, it was shown that PvGAMA consists of two conserved binding fragments that bind preferentially to most immature human reticulocytes, which is consistent with the $P$. vivax invasion phenotype and highlights the fact that functional regions can be predicted by analysing natural selection. Further studies aimed at discerning the function of conserved regions as vaccine components are required.

\section{Additional files}

\begin{abstract}
Additional file 1: Figure S1. GAMA antigen alignment. pvgama sequences from 6 P. vivax strains were aligned with orthologous sequences from P. cynomolgi, P. inui, P. fragile, P. coatneyi and P. knowlesi. a DNA sequence alignment. b Deduced amino acid alignment. The sequences were obtained from GenBank: access numbers being India-VII AFBK01000586-AFBK01000587, North Korean AFNJ01000531, Brazil-I AFMK01000508-AFMK01000509, Mauritania-I AFNI01000333-

AFNI01000334, P. inui NW_0084818881, P. fragile NW_012192586, P. cynomolgi BAEJ01000249, P. coatneyi CM0028561 and P. knowlesi NC_0119061. (PDF $373 \mathrm{~kb})$
\end{abstract}

Additional file 2: Figure S2. Lineage-specific positive selection. Branches under positive episodic selection were identified by using the REL-site branch method. Episodic selection acts very quickly and involves a switch from negative to positive natural selection and back to negative and might enable adaptation to a new host. Phylogeny was inferred in MEGA v6 by the maximum likelihood method using the $G T R+G$ evolutionary model. $\omega^{+}$model: $\omega$ rate values. $\operatorname{Pr}[\omega=\omega+]$ : percentage of sites evolving under positive selection. $P$-value corrected for multiple tests using the Holm-Bonferroni method. (TIF $470 \mathrm{~kb}$ )

Additional file 3: Figure S3. Obtaining recombinant proteins. a, b Recombinant GAMA protein expression and purification. Lanes 2-3 show non-induced and induced cell lysate, respectively. Lanes 4-5 show purified rPVGAMA-Nt and -Ct stained with Coomassie blue or analysed by western blot using anti-polyhistidine antibodies, respectively. c Purifying conserved (CR1 and CR2) and variable (VR1 and VR2) PVGAMA regions. Lanes 2, 4, 6 and 8 show purified recombinant proteins and lanes 3, 5, 7 and 9 show western blot detection. The proteins' molecular markers are indicated in Lane 1 on all figures. (TIF $5327 \mathrm{~kb}$ )

Additional file 4: Figure S4. Selection strategy for immature and mature erythrocyte populations. The doublets were excluded by plotting FSC-H against FSC-A. Cells were then selected by their granularity, using an SSC-A vs FSC-A cytogram. The CD45 vs CD71 signal was plotted for selecting reticulocyte (CD71 + CD45-) and mature erythrocyte (CD71-CD45-) populations and omitting activated lymphocytes (CD71 + CD45+). The percentage of cells having bound protein was calculated using the PE signal $(C D 71+C D 45-P E+)$. A representative histogram from three independent experiments analysing the PE signal for the CR2 binding assay compared to control is also shown. (TIF $10448 \mathrm{~kb}$ )

\section{Abbreviations}

ANOVA: Analysis of variance; $C D 71^{\text {hi }}$ : $C D 71$ high; $C D 71^{\text {lo }}$ : CD71 low; CR: Conserved region; DARC: Duffy antigen receptor for chemokines; DBP: Duffy binding protein; DEB: Denaturing extraction buffer; EBP: Erythrocyte binding protein; ELISA: Enzyme-linked immunosorbent assay; LB: Luria bertani; MALDI-TOF: Matrix-assisted laser desorption/ ionization-time of flight; MS: Mass spectrometry; MSP-1: Merozoite surface protein 1; NEB: Native extraction buffer; OD: Optical density; PBS: Phosphate buffered saline; PVGAMA: P. vivax GPI-anchored micronemal antigen; Pvgama: Plasmodium vivax gama; RBP: Reticulocyte binding protein; RON5: Rhoptry neck protein 5; RP-HPLC: Reverse phase high-performance liquid chromatography; RR: Repeat region; RT: Room temperature;

SD: Standard deviation; TRAg: Tryptophan-rich antigen; VCG-I: Vivax Colombia Guaviare 1; VR: Variable region

\section{Acknowledgements}

We would like to thank Ana María Perdomo and Bernardo Camacho for supplying the umbilical cord blood, Diana Díaz for technical support in cytometry and Jason Garry for translating this manuscript.

\section{Funding}

This research was financed by the Colombian Science, Technology and Innovation Department (COLCIENCIAS), contract RC\#0309-2013. During the course of this research JFR and HDOS were financed via COLCIENCIAS cooperation agreement \# 0719-2013 and \# 0555-2015, respectively. The sponsors had no role in study design, collection and analysis or data interpretation. 


\section{Availability of data and materials}

All data generated or analysed during this study are included within this article and its additional files. The pvgama sequence from P. vivax VCG-I strain was deposited in the GenBank database under accession number KT248546.

\section{Authors' contributions}

$\angle A B$ and DAMP devised and designed the study; $L A B, D A M P, D G O$, JFR and HDOS performed the experiments; $L A B, D A M P, D G O$ and MAP analysed the results and wrote the manuscript. All authors read and approved the final manuscript.

\section{Competing interests}

The authors declare that they have no competing interests.

\section{Consent for publication}

Not applicable.

\section{Ethics approval and consent to participate}

All individuals who participated in this research (including progenitors regarding umbilical cord samples) signed an informed consent form after receiving detailed information regarding the study's goals. All procedures were approved by FIDIC's ethics committee.

\section{Publisher's Note}

Springer Nature remains neutral with regard to jurisdictional claims in published maps and institutional affiliations.

\section{Author details \\ ${ }^{1}$ Molecular Biology and Immunology Department, Fundación Instituto de Inmunología de Colombia (FIDIC), Carrera 50 No. 26-20, Bogotá DC, Colombia. ${ }^{2} \mathrm{PhD}$ Programme in Biomedical and Biological Sciences, Universidad del Rosario, Carrera 24 No. 63C-69, Bogotá DC, Colombia. ${ }^{3}$ Basic Sciences Department, School of Medicine and Health Sciences, Universidad del Rosario, Carrera 24 No. 63C-69, Bogotá DC, Colombia.}

\section{Received: 27 October 2016 Accepted: 10 May 2017}

\section{Published online: 19 May 2017}

\section{References}

1. WHO. World malaria report. 2014.

2. WHO. State of the art of new vaccine research and development. 2006.

3. Patarroyo ME, Bermudez A, Patarroyo MA. Structural and immunological principles leading to chemically synthesized, multiantigenic, multistage, minimal subunit-based vaccine development. Chem Rev. 2011;111:3459-507.

4. Lanzavecchia A, Fruhwirth A, Perez L, Corti D. Antibody-guided vaccine design: identification of protective epitopes. Curr Opin Immunol. 2016;41:62-7.

5. Gardner MJ, Hall N, Fung E, White O, Berriman M, Hyman RW, et al. Genome sequence of the human malaria parasite Plasmodium falciparum. Nature. 2002;419:498-511.

6. Bozdech Z, Llinas M, Pulliam BL, Wong ED, Zhu J, DeRisi JL. The transcriptome of the intraerythrocytic developmental cycle of Plasmodium falciparum. PLoS Biol. 2003;1:E5

7. Lasonder E, Ishihama Y, Andersen JS, Vermunt AM, Pain A, Sauerwein RW, et al. Analysis of the Plasmodium falciparum proteome by high-accuracy mass spectrometry. Nature. 2002;419:537-42.

8. Cowman AF, Berry D, Baum J. The cellular and molecular basis for malaria parasite invasion of the human red blood cell. J Cell Biol. 2012;198:961-71.

9. Richards JS, Beeson JG. The future for blood-stage vaccines against malaria. Immunol Cell Biol. 2009;87:377-90.

10. Arumugam TU, Takeo S, Yamasaki T, Thonkukiatkul A, Miura K, Otsuki H, et al. Discovery of GAMA, a Plasmodium falciparum merozoite micronemal protein as a novel blood-stage vaccine candidate antigen. Infect Immun. 2011;79: 4523-32.

11. Huynh MH, Carruthers VB. A Toxoplasma gondii Ortholog of Plasmodium GAMA contributes to parasite attachment and cell invasion. mSphere. 2016;1: doi:10.1128/mSphere.00012-16.

12. Moreno-Perez DA, Areiza-Rojas R, Florez-Buitrago X, Silva $Y$, Patarroyo ME, Patarroyo MA. The GPI-anchored 6-Cys protein PV12 is present in detergentresistant microdomains of Plasmodium vivax blood stage schizonts. Protist. 2013;164:37-48
13. Patarroyo MA, Calderón D, Moreno-Pérez DA. Vaccines against Plasmodium vivax: a research challenge. Expert Rev Vaccines. 2012;11:1249-60.

14. Urquiza M, Patarroyo MA, Mari V, Ocampo M, Suarez J, Lopez R, et al. Identification and polymorphism of Plasmodium vivax RBP-1 peptides which bind specifically to reticulocytes. Peptides. 2002;23:2265-77.

15. Franca CT, He WQ, Gruszczyk J, Lim NT, Lin E, Kiniboro B, et al. Plasmodium vivax reticulocyte binding proteins are key targets of naturally acquired immunity in young Papua New Guinean children. PLoS Negl Trop Dis. 2016;10:e0005014.

16. Rodriguez LE, Urquiza M, Ocampo M, Curtidor H, Suarez J, Garcia J, et al. Plasmodium vivax MSP-1 peptides have high specific binding activity to human reticulocytes. Vaccine. 2002;20:1331-9.

17. Zeeshan M, Tyagi RK, Tyagi K, Alam MS, Sharma YD. Host-parasite interaction: selective PV-fam-a family proteins of Plasmodium vivax bind to a restricted number of human erythrocyte receptors. J Infect Dis. 2015;211:1111-20.

18. Arevalo-Pinzon G, Bermudez M, Curtidor H, Patarroyo MA. The Plasmodium vivax rhoptry neck protein 5 is expressed in the apical pole of Plasmodium vivax VCG-1 strain schizonts and binds to human reticulocytes. Malaria J. 2015;14:106.

19. Garzon-Ospina D, Forero-Rodriguez J, Patarroyo MA. Inferring natural selection signals in Plasmodium vivax-encoded proteins having a potential role in merozoite invasion. Infect Genet Evol. 2015;33:182-8.

20. Cornejo OE, Fisher D, Escalante AA. Genome-wide patterns of genetic polymorphism and signatures of selection in Plasmodium vivax. Genome Biol Evol. 2014;7:106-19.

21. Neafsey DE, Galinsky K, Jiang RH, Young L, Sykes SM, Saif S, et al. The malaria parasite Plasmodium vivax exhibits greater genetic diversity than Plasmodium falciparum. Nat Genet. 2012;44:1046-50.

22. Forero-Rodriguez J, Garzon-Ospina D, Patarroyo MA. Low genetic diversity and functional constraint in loci encoding Plasmodium vivax P12 and P38 proteins in the Colombian population. Malaria J. 2014;13:58.

23. Forero-Rodriguez J, Garzon-Ospina D, Patarroyo MA. Low genetic diversity in the locus encoding the Plasmodium vivax P41 protein in Colombia's parasite population. Malaria J. 2014;13:388.

24. Wang Y, Ma A, Chen SB, Yang YC, Chen JH, Yin MB. Genetic diversity and natural selection of three blood-stage 6-Cys proteins in Plasmodium vivax populations from the China-Myanmar endemic border. Infect Genet Evol. 2014;28:167-74.

25. Graur D, Zheng Y, Price N, Azevedo RB, Zufall RA, Elhaik E. On the immortality of television sets: "function" in the human genome according to the evolution-free gospel of ENCODE. Genome Biol Evol. 2013;5:578-90.

26. Escalante AA, Cornejo OE, Freeland DE, Poe AC, Durrego E, Collins WE, et al. A monkey's tale: the origin of Plasmodium vivax as a human malaria parasite. Proc Natl Acad Sci USA. 2005;102:1980-5.

27. Edgar RC. MUSCLE: multiple sequence alignment with high accuracy and high throughput. Nucleic Acids Res. 2004;32:1792-7.

28. Zhang J, Rosenberg HF, Nei M. Positive Darwinian selection after gene duplication in primate ribonuclease genes. Proc Natl Acad Sci USA. 1998;95: 3708-13.

29. Jukes THaCRC. Evolution of protein molecules. In: Munro HN, editor Mammalian protein metabolism. New York: Academic Press; 1969.

30. Kosakovsky Pond SL, Frost SD. Not so different after all: a comparison of methods for detecting amino acid sites under selection. Mol Biol Evol. 2005;22:1208-22.

31. Murrell B, Wertheim JO, Moola S, Weighill T, Scheffler K, Kosakovsky Pond SL. Detecting individual sites subject to episodic diversifying selection. PLoS Genet. 2012;8:e1002764.

32. Murrell B, Moola S, Mabona A, Weighill T, Sheward D, Kosakovsky Pond SL, et al. FUBAR: a fast, unconstrained bayesian approximation for inferring selection. Mol Biol Evol. 2013;30:1196-205.

33. Kosakovsky Pond SL, Posada D, Gravenor MB, Woelk CH, Frost SD. Automated phylogenetic detection of recombination using a genetic algorithm. Mol Biol Evol. 2006;23:1891-901.

34. Kosakovsky Pond SL, Murrell B, Fourment M, Frost SD, Delport W, Scheffler K. A random effects branch-site model for detecting episodic diversifying selection. Mol Biol Evol. 2011;28:3033-43.

35. Delport W, Poon AF, Frost SD, Kosakovsky Pond SL. Datamonkey 2010: a suite of phylogenetic analysis tools for evolutionary biology. Bioinformatics. 2010:26:2455-7.

36. Aurrecoechea C, Brestelli J, Brunk BP, Dommer J, Fischer S, Gajria B, et al. PlasmoDB: a functional genomic database for malaria parasites. Nucleic Acids Res. 2009;37:D539-543. 
37. Moreno-Perez DA, Degano R, Ibarrola N, Muro A, Patarroyo MA. Determining the Plasmodium vivax VCG-1 strain blood stage proteome. J Proteomics. 2014;113C:268-80.

38. Pico de Coana Y, Rodriguez J, Guerrero E, Barrero C, Rodriguez R, Mendoza $M$, et al. A highly infective Plasmodium vivax strain adapted to Aotus monkeys: quantitative haematological and molecular determinations useful for $P$. vivax malaria vaccine development. Vaccine. 2003;21:3930-7.

39. Thompson JD, Higgins DG, Gibson TJ. CLUSTAL W: improving the sensitivity of progressive multiple sequence alignment through sequence weighting, position-specific gap penalties and weight matrix choice. Nucleic Acids Res. 1994;22:4673-80.

40. Houghten RA. General method for the rapid solid-phase synthesis of large numbers of peptides: specificity of antigen-antibody interaction at the level of individual amino acids. Proc Natl Acad Sci USA. 1985;82:5131-5.

41. Lu F, Li J, Wang B, Cheng Y, Kong DH, Cui L, et al. Profiling the humoral immune responses to Plasmodium vivax infection and identification of candidate immunogenic rhoptry-associated membrane antigen (RAMA). J Proteomics. 2014;102C:66-82.

42. Ntumngia FB, Thomson-Luque R, Torres Lde M, Gunalan K, Carvalho LH, Adams $\mathrm{JH}$. A novel erythrocyte binding protein of Plasmodium vivax suggests an alternate invasion pathway into Duffy-positive reticulocytes. mBio. 2016;7: doi: 10.1128/mBio.01261-16

43. Moreno-Perez DA, Saldarriaga A, Patarroyo MA. Characterizing PvARP, a novel Plasmodium vivax antigen. Malaria J. 2013;12:165.

44. Mongui A, Angel DI, Gallego G, Reyes C, Martinez P, Guhl F, et al. Characterization and antigenicity of the promising vaccine candidate Plasmodium vivax 34 kDa rhoptry antigen (PV34). Vaccine. 2009;28:415-21.

45. Moreno-Perez DA, Montenegro M, Patarroyo ME, Patarroyo MA. Identification, characterization and antigenicity of the Plasmodium vivax rhoptry neck protein 1 (PVRON1). Malaria J. 2011;10:314.

46. Arevalo-Pinzon G, Curtidor H, Patino LC, Patarroyo MA. PVRON2, a new Plasmodium vivax rhoptry neck antigen. Malaria J. 2011;10:60.

47. Arevalo-Pinzon G, Curtidor H, Abril J, Patarroyo MA. Annotation and characterization of the Plasmodium vivax rhoptry neck protein 4 (PVRON4). Malaria J. 2013;12:356.

48. Buitrago SP, Garzon-Ospina D, Patarroyo MA. Size polymorphism and low sequence diversity in the locus encoding the Plasmodium vivax rhoptry neck protein 4 (PVRON4) in Colombian isolates. Malaria J. 2016:15:501.

49. Garzon-Ospina D, Forero-Rodriguez J, Patarroyo MA. Evidence of functional divergence in MSP7 paralogous proteins: a molecular-evolutionary and phylogenetic analysis. BMC Evol Biol. 2016;16:256.

50. Muehlenbein MP, Pacheco MA, Taylor JE, Prall SP, Ambu L, Nathan S, et al. Accelerated diversification of nonhuman primate malarias in Southeast Asia: adaptive radiation or geographic speciation? Mol Biol Evol. 2015;32:422-39.

51. Sawai H, Otani H, Arisue N, Palacpac N, de Oliveira ML, Pathirana S, et al. Lineage-specific positive selection at the merozoite surface protein 1 (msp1) locus of Plasmodium vivax and related simian malaria parasites. BMC Evol Biol. 2010;10:52.

52. Schofield $\mathrm{L}$. On the function of repetitive domains in protein antigens of Plasmodium and other eukaryotic parasites. Parasitol Today. 1991;7:99-105.

53. Ferreira MU, da Silva NM, Wunderlich G. Antigenic diversity and immune evasion by malaria parasites. Clin Diagn Lab Immunol. 2004;11:987-95.

54. Patarroyo ME, Patarroyo MA. Emerging rules for subunit-based, multiantigenic, multistage chemically synthesized vaccines. Acc Chem Res. 2008;41:377-86.

55. Martinez P, Suarez CF, Gomez A, Cardenas PP, Guerrero JE, Patarroyo MA. High level of conservation in Plasmodium vivax merozoite surface protein 4 (PvMSP4). Infect Genet Evol. 2005;5:354-61.

56. Putaporntip C, Jongwutiwes $S$, Ferreira MU, Kanbara $H$, Udomsangpetch $R$, Cui L. Limited global diversity of the Plasmodium vivax merozoite surface protein 4 gene. Infect Genet Evol. 2009;9:821-6.

57. Garzon-Ospina D, Romero-Murillo L, Tobon LF, Patarroyo MA. Low genetic polymorphism of merozoite surface proteins 7 and 10 in Colombian Plasmodium vivax isolates. Infect Genet Evol. 2011;11:528-31.

58. Garzon-Ospina D, Forero-Rodriguez J, Patarroyo MA. Heterogeneous genetic diversity pattern in Plasmodium vivax genes encoding merozoite surface proteins (MSP) -7E, -7 F and -7 L. Malaria J. 2014;13:495

59. Pacheco MA, Elango AP, Rahman AA, Fisher D, Collins WE, Barnwell JW, et al. Evidence of purifying selection on merozoite surface protein 8 (MSP8) and 10 (MSP10) in Plasmodium spp. Infect Genet Evol. 2012;12:978-86.
60. Garzon-Ospina D, Romero-Murillo L, Patarroyo MA. Limited genetic polymorphism of the Plasmodium vivax low molecular weight rhoptry protein complex in the Colombian population. Infect Genet Evol. 2010;10:261-7.

61. Cheng Y, Lu F, Wang B, Li J, Han JH, Ito D, et al. Plasmodium vivax GPI-anchored micronemal antigen (PVGAMA) binds human erythrocytes independent of Duffy antigen status. Sci Reports. 2016;6:35581.

62. Malleret B, Li A, Zhang R, Tan KS, Suwanarusk R, Claser C, et al. Plasmodium vivax: restricted tropism and rapid remodeling of CD71-positive reticulocytes. Blood. 2015;125:1314-24.

63. Wilson MC, Trakarnsanga K, Heesom KJ, Cogan N, Green C, Toye AM, et al. Comparison of the proteome of adult and cord erythroid cells, and changes in the proteome following reticulocyte maturation. Mol Cel Proteomics MCP. 2016;15:1938-46.

64. Ocampo M, Vera R, Eduardo R, Curtidor H, Urquiza M, Suarez J, et al. Plasmodium vivax Duffy binding protein peptides specifically bind to reticulocytes. Peptides. 2002;23:13-22.

65. Han JH, Lee SK, Wang B, Muh F, Nyunt MH, Na S, et al. Identification of a reticulocyte-specific binding domain of Plasmodium vivax reticulocytebinding protein 1 that is homologous to the PfRh4 erythrocyte-binding domain. Sci Reports. 2016;6:26993.

66. Dent $A E$, Nakajima R, Liang L, Baum E, Moormann AM, Sumba PO, et al. Plasmodium falciparum protein microarray antibody profiles correlate with protection from symptomatic malaria in Kenya. J Infect Dis. 2015;212:1429-38.

\section{Submit your next manuscript to BioMed Central and we will help you at every step:}

- We accept pre-submission inquiries

- Our selector tool helps you to find the most relevant journal

- We provide round the clock customer support

- Convenient online submission

- Thorough peer review

- Inclusion in PubMed and all major indexing services

- Maximum visibility for your research

Submit your manuscript at www.biomedcentral.com/submit 